\title{
Use of Sample Bias Voltage for Low-Energy High-Resolution Imaging in the SEM
}

\author{
J.R. Michael ${ }^{1}$, D. C. Joy ${ }^{2}$ and B. J. Griffin ${ }^{3}$ \\ ${ }^{1}$ Sandia National Laboratories, PO Box 5800, Albuquerque, NM 87185-0886 \\ ${ }^{2}$ Center for NanoPhase Materials Science, Oak Ridge National Laboratory, Oak Ridge, \\ TN 37831 \\ ${ }^{3}$ Centre for Microscopy, Characterization and Analysis, The University of Western Australia, \\ Crawley, WA Australia 6009
}

Low beam voltage operation of the SEM has been shown to produce secondary electron images with high resolution.[1] The ability to form small electron probes is limited in practice by the initial probe size, spherical aberration and chromatic aberration. At low voltages, chromatic aberration due to the spread of electron energies emitted by the electron source, dominates the probe size and limits the image resolution of low voltage secondary electron imaging. The energy spread of the electron source can be reduced through the use of room temperature field emission (FE) sources or a monochromator may be employed to filter the electron beam to reduce the energy spread. The combination of a thermal or Schottky FE source with a monochromator preserves the high beam current capability of the thermal FE source while allowing lowered energy spreads in the electron probe and reduced chromatic aberrations. Final image resolution at low voltages is very dependent upon the quality of the electron column and there are lower limits to the electron beam voltage imposed by the design of the electron source and column. The application of sample or stage bias to decelerate the electron beam before interaction with the sample can produce high resolution images at very low beam voltages.[2] The use of sample bias, or cathode lens mode, reduces the aberration coefficients of the electron column by the ratio of the energy in the column to the landing energy of the beam (column energy- sample bias) thus allowing high resolution images to be obtained.[2]

An FEI Magellan 400 SEM was used in these studies. This instrument is equipped with a fixed energy spread monochromator in the electron gun that limits the energy spread of the electron beam to less than $0.2 \mathrm{ev}$ and is operable when the primary beam voltage is $5 \mathrm{kV}$ or less. The monochromator was used for this work. Previous work has shown this SEM can produce images with resolutions of better than $1 \mathrm{~nm}$ at $1 \mathrm{kV}$.[1] A sample bias of 50 to $4000 \mathrm{v}$ can be applied to the stage to decelerate the electron beam. The results shown here are images at landing voltages of $400 \mathrm{v}$ and $1000 \mathrm{v}$ of ion sputtered Pt islands which were obtained by varying the voltage of the electron gun and the applied sample bias. Image resolution was measured using the contrast transfer function.[3]

Figure 1 was obtained at a beam voltage of $5 \mathrm{kV}$ and a sample bias of $4 \mathrm{kV}$ resulting in a landing energy of $1 \mathrm{kV}$. There is very good image detail visible. Figure 2 is similar to Figure 1 except that the beam voltage was $4.4 \mathrm{kV}$ and the sample bias was $4 \mathrm{kV}$ resulting in a landing energy of $400 \mathrm{v}$. Figure 3 and 4 are the contrast transfer functions (CTF) of the images shown in figures 1 and 2 respectively.[1] The CTF shows that the resolution at the point when the $\mathrm{S} / \mathrm{N}$ reaches 1 is $1.1 \mathrm{~nm}$ for a landing energy of $1 \mathrm{kV}$ and 1.0 for a landing energy of $400 \mathrm{v}$. These results demonstrate the advantages of using the beam deceleration mode of operation. The resolution at low voltages is quite good and does not degrade rapidly with decreasing beam voltage as would be expected from normal operation without beam deceleration due to the reduction in the aberration coefficients 
proportional to the ratio of the landing energy and the beam energy. These examples show the advantage of using sample bias with a flat sample with little surface topography. Sample topography or charging of non-conductive regions can distort the focusing fields introduced by the use of sample bias and can result in uncorrectable image distortions. The application of sample bias with the right samples can provide very high resolution images in the SEM at low landing voltages.

References :

[1] J. R. Michael et al. Microsc. Microanal, 15(suppl 2) (2009) 660CD

[2] I. Mullerova et al., Scanning, 15(1993) 193..

[3] D. C. Joy et al., Microsc. Microanal, 13(suppl 2) (2007) 1682CD

[4] Sandia is a multiprogram laboratory operated by Sandia Corporation, a Lockheed Martin Company, for the United States Department of Energy (DOE) under contract DEAC0494AL85000.
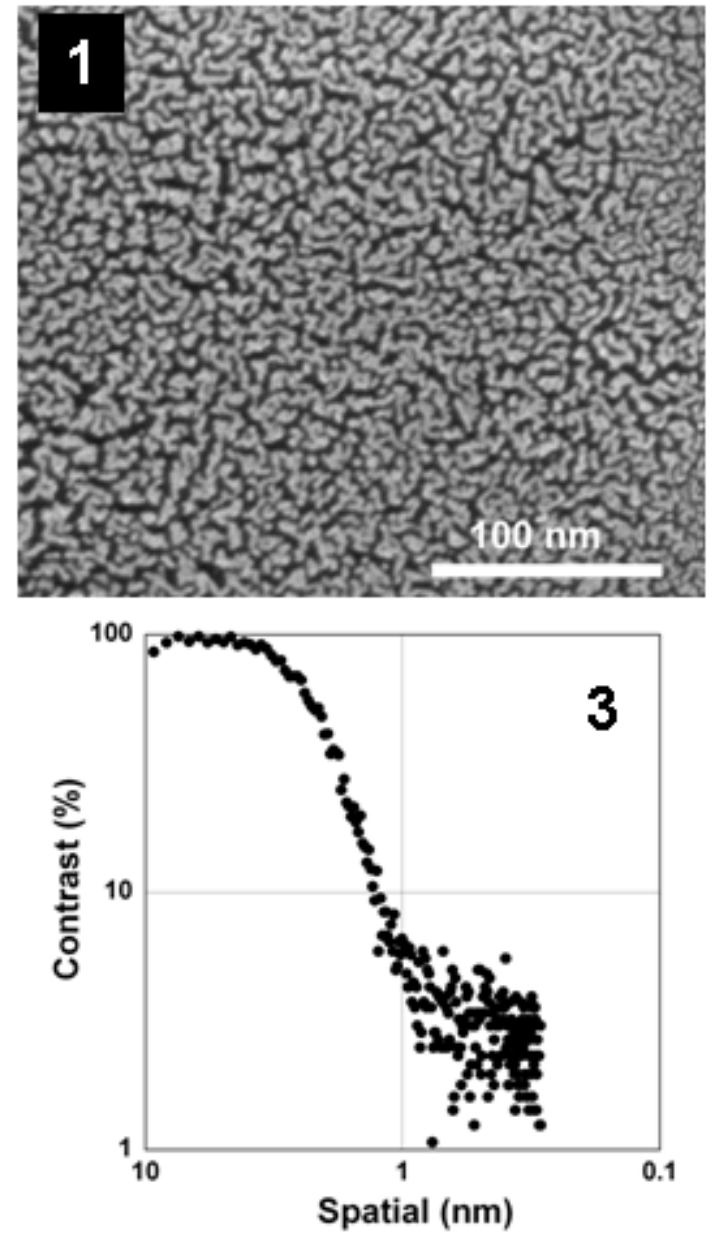
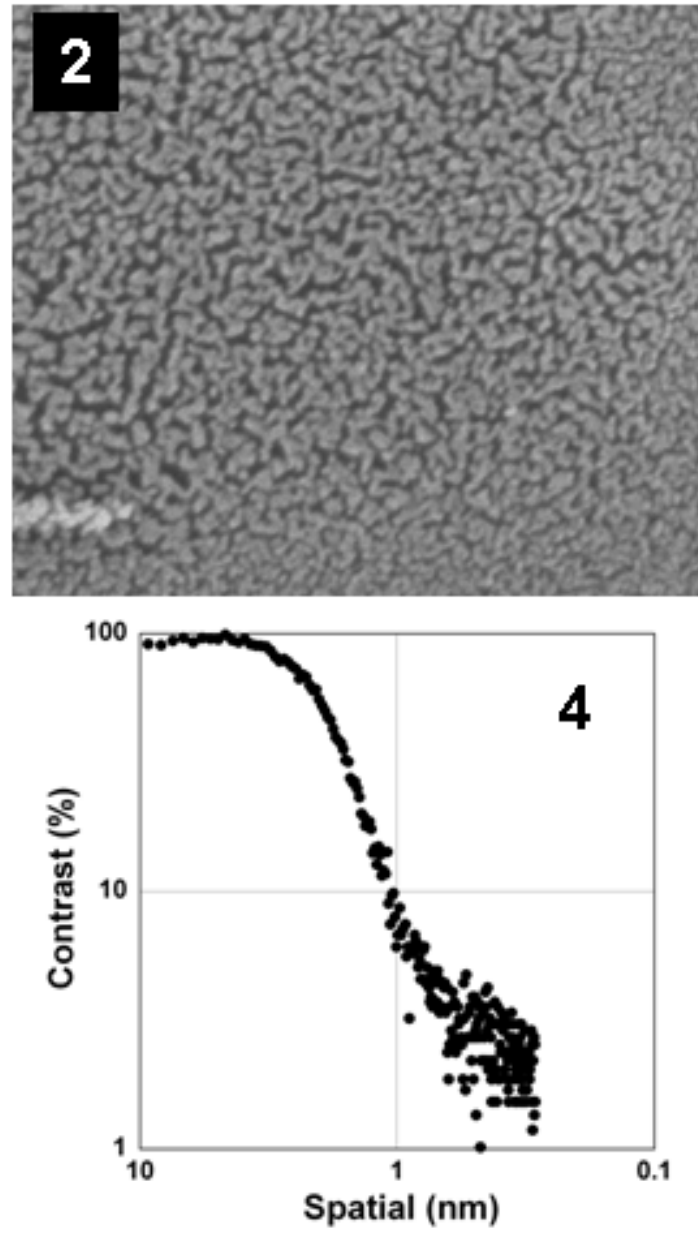

Figure 1. Ion sputtered Pt imaged with a landing energy of $1 \mathrm{kV}$ and a bias of $4 \mathrm{kV}$. Figure 2. Ion sputtered Pt imaged with a landing energy of $400 \mathrm{~V}$ and a bias of $4 \mathrm{kV}$. Figure 3. CTF calculated from Fig. 1 that shows contrast transfer down to $1.1 \mathrm{~nm}$. Figure 4. CTF calculated from Fig. 2 that shows contrast transfer down to $1.0 \mathrm{~nm}$. 